\title{
Nurse home visiting and prenatal substance use in a socioeconomically disadvantaged population in British Columbia: analysis of prenatal secondary outcomes in an ongoing randomized controlled trial
}

\author{
Nicole L.A. Catherine PhD, Michael Boyle PhD, Yufei Zheng MPH, Lawrence McCandless PhD, \\ Hui Xie PhD, Rosemary Lever MA, Debbie Sheehan MSW, Andrea Gonzalez PhD, \\ Susan M. Jack RN PhD, Amiram Gafni PhD, Lil Tonmyr PhD, Lenora Marcellus RN PhD, \\ Colleen Varcoe RN PhD, Ange Cullen MPH, Kathleen Hjertaas BES, Caitlin Riebe BSc, \\ Nikolina Rikert BA, Ashvini Sunthoram BSc, Ronald Barr MDCM, Harriet MacMillan MD, \\ Charlotte Waddell MD
}

\section{Abstract}

Background: Nurse-Family Partnership (NFP) involves public health nurses providing frequent home visits from early pregnancy until children reach age 2 years, focusing on first-time parents experiencing socioeconomic disadvantage. Our aim was to evaluate NFP's effectiveness in improving child and maternal health.

\begin{abstract}
Methods: We conducted an analysis of prenatal secondary outcomes in an ongoing randomized controlled trial in British Columbia; the data used in this analysis were collected from January 2014 to May 2017. Participants were pregnant girls and women aged 14-24 years who were preparing to parent for the first time and experiencing socioeconomic disadvantage. They were randomly allocated 1:1 to the intervention (NFP plus existing services) or control group (existing services). Prespecified prenatal secondary outcome indicators were changes in use of nicotine cigarettes and alcohol use by 34-36-weeks' gestation. We also report on prespecified exploratory cannabis and street drug use measures. We used mixed-effect models for longitudinal and clustered data to estimate intervention effects. Analyses were by intention to treat.
\end{abstract}

Results: The median gestational age at baseline for the 739 participants (368 participants in the intervention group, 371 in the comparison group) was 20 weeks, 6 days. By 34-36 weeks' gestation, NFP significantly reduced cigarette counts (over the past 2 d) (difference in changes [DIC] of count $-1.6,95 \%$ confidence interval $[\mathrm{Cl}]-6.4$ to -1.3 ) in those who smoked. NFP also significantly reduced rates of prenatal cannabis use (DIC $-6.4,95 \% \mathrm{Cl}-17.0$ to -1.7$)$, but not rates of street drug or "any" substance use. While we observed decreased rates of cigarette and alcohol use in both groups (DIC of proportions $-2.8,95 \% \mathrm{Cl}-15.3$ to 0.6 ; DIC -0.5 , $95 \% \mathrm{Cl}-8.7$ to 1.8 , respectively), these changes were not statistically significant.

Interpretation: We found no evidence that NFP was effective in reducing rates of prenatal cigarette and alcohol use; however, it led to reduced prenatal cannabis use, and in smokers it led to modest reductions in cigarette use. NFP may therefore hold promise for reducing some types of prenatal substance use in disadvantaged populations. Trial registration: ClinicalTrials.gov, no. NCT01672060.

$\mathrm{P}$ renatal exposures to nicotine cigarettes (hereafter cigarettes), alcohol, cannabis, cocaine and opioids put children at risk for adverse outcomes including preterm birth, low birth weight, motor abnormalities, and mental health and cognitive problems. ${ }^{1-9}$ Rates of prenatal substance use in Canada remain concerning, particularly for cigarettes $(23 \%),{ }^{10}$ alcohol $(10 \%)^{11}$ and cannabis $(7 \%) .{ }^{12}$ Accordingly, efforts to prevent prenatal substance use are warranted, ${ }^{13-15}$ particularly among people who are young, living on low income and coping with mental health challenges and are therefore at higher risk of prenatal substance use. ${ }^{16}$ New research is needed on effective interventions to reduce prenatal substance use. . $^{10,14,17-19}$

Competing interests: None declared.

This article has been peer reviewed.

Correspondence to: Charlotte Waddell, charlotte_waddell@sfu.ca CMAJ Open 2020. DOI:10.9778/cmajo.20200063 
Aiming to improve child and maternal well-being, NurseFamily Partnership (NFP) involves public health nurses providing intensive home visits to first-time expectant parents experiencing socioeconomic disadvantage..$^{20}$ Randomized controlled trials (RCTs) in the United States have shown that NFP improves child mental health and cognitive development and reduces child injuries by age 2 years, while also improving maternal well-being. ${ }^{20}$ However, NFP's effectiveness in reducing prenatal substance use remains unclear. ${ }^{21,22}$ It also remains unknown how NFP's benefits may translate to Canada, given the greater availability of publicly funded health and social services in this country compared with the US.

The British Columbia Healthy Connections Project RCT aims to address these evidence gaps. ${ }^{23,24}$ The trial is ongoing (2011-2022). We report here on NFP's impact on prenatal cigarette and alcohol use, which are prespecified secondary outcome indicators. We also report prespecified exploratory data on program effects for prenatal use of cannabis and street drugs. Reports on additional child and maternal outcomes will follow.

\section{Methods}

\section{Setting}

This ongoing, single-blind RCT is being conducted in 26 urban and suburban local health areas across 4 of the 5 $\mathrm{BC}$ regional health authorities (Appendix 1A, available at www.cmajopen.ca/content/8/4/E667/suppl/DC1).

\section{Design, recruitment and population}

This is an analysis of prespecified RCT prenatal secondary outcome indicators. For the RCT, health authorities were responsible for recruiting pregnant girls and young women. They contacted primary care providers and community agencies to encourage referrals to public health units, where staff screened referrals then passed information on potential participants to the study team. The study team then contacted potential participants to introduce the RCT, confirm eligibility and schedule baseline interviews.

The inclusion criteria for the RCT focused on girls and young women who were preparing to parent for the first time, were experiencing socioeconomic disadvantage and were aged 24 years or younger - the population most likely to benefit from NFP (Box 1). ${ }^{20}$

Recruitment of participants for the RCT started in October 2013 and closed in December 2016. Prenatal data collection concluded in May 2017. Intervention participants completed NFP in June 2019. We finished prenatal analyses in August 2019 and completed all postpartum research interviews in November 2019. Administrative data collection on child injuries will continue into 2021.

\section{Randomization procedures}

For the RCT, following baseline interviews, participants were randomly assigned (1:1) to either the intervention group (NFP plus existing services) or the comparison group (existing services). We used an unpredictable, randomized sequence protocol developed by an independent statistician, applying a separate randomization schedule for all 26 local health areas. We used constrained randomization, assigning the smaller of 2 block sizes where 17 or fewer participants were expected annually. An investigator who was uninvolved in data collection performed computerized allocation and informed participants and NFP nurses of treatment allocation (i.e., unmasked treatment allocation). Field interviewers collecting data were masked to group allocation; they also reminded participants before each interview not to reveal their group. Trial statisticians and methodologists masked to group assignment conducted the main analyses.

\section{Intervention}

For the trial, nurses were recruited by health authorities then completed NFP education comprising approximately 140 classroom hours and ongoing learning. ${ }^{25}$ (Nurses consented to participate in the RCT and received research orientations.) After participants were allocated to a study group, nurses contacted those allocated to the intervention group to schedule initial visits and offer to deliver NFP. According to

Box 1: Inclusion and exclusion criteria at time of baseline interview

Inclusion criteria

1. Age 24 years or younger*

2. Preparing to parent for the first time†

3. Pregnant and less than 28 weeks' gestation

4. Competent to provide informed consent, including conversational in English§

5. Experiencing socioeconomic disadvantage

- Age 19 years or younger

- Age 20-24 years and meets 2 of the following 3 indicators: (a) lone parent, $\emptyset$ (b) less than grade $12,{ }^{* *}$ (c) low income (1 or more of the following):

- Receiving income assistance

- Finding it very difficult to live on total household income regarding food or rent

- Homeless, defined as living on the streets, in an emergency or homeless shelter, staying in places not meant as residences (e.g., car or tent) or experiencing "hidden homelessness" such as "couch surfing"

\section{Exclusion criteria}

- Planning to have the child adopted

- Planning to leave the catchment area for 3 months or longer during the trialt†

Note: Criteria were previously reported by Catherine et al.23.24

*No lower age limit was set.

†Eligible if a previous pregnancy ended in termination, miscarriage or stillbirth or if previous parenting involved step-parenting only.

$\ddagger$ Must receive first Nurse-Family Partnership (NFP) visit by end of 28th week of gestation, according to NFP fidelity requirements.

$\S$ Must be able to participate without an interpreter.

INot married or living with the same partner for 1 year or more consecutively.

**Did not complete secondary school or did not receive secondary school equivalency certificate.

† Catchment refers to designated $\mathrm{BC}$ local health areas offering the $\mathrm{BC}$ Healthy Connections Project. 
NFP guidelines, there could be as many as 14 visits prenatally and 50 postpartum. Visits followed NFP guidelines, with nurses assisting participants to identify and meet health and social goals, including but not limited to reducing prenatal substance use. ${ }^{25}$ Nurses also received intensive supervision to ensure program fidelity. (Copyrighted NFP materials were accessed through a BC Ministry of Health licence. ${ }^{25}$ )

\section{Existing services}

Participants in both the intervention and comparison groups could access existing prenatal health and social services. These included primary or specialist health care or both; hospital or emergency care or both; mental health services, including services for problematic substance use; public health services, including prenatal classes; and social and community programs.

\section{Data sources}

All eligible participants provided written informed consent before starting the study. A series of validated scales and items were administered at baseline (before 28 weeks' gestation; in person) and in late pregnancy (at 34-36 weeks' gestation; telephone) to collect information on sociodemographic characteristics (Statistics Canada census), ${ }^{26}$ psychological distress (10-item Kessler Psychological Distress Scale), ${ }^{27}$ experiences of violence (Childhood Trauma Questionnaire, Composite Abuse Scale) ${ }^{28,29}$ and prenatal substance use (Statistics Canada National Longitudinal Survey of Children and Youth) ${ }^{30}$ (Table 1). Following each interview, participants received gift cards (\$50-\$75, depending on interview duration) for local stores. Field interviewers verbally administered questionnaires. For items prone to response bias (including items on prenatal substance use), participants responded confidentially on paper. Participants could decline to answer questions. Participants also chose the locations for in-person interviews; interviews were usually conducted in their homes, but private rooms at local libraries or community centres were used when privacy or housing instability was a concern.

\section{Outcome indicators}

The prespecified main trial outcome indicators were as follows: child injuries by age 2 years (primary indicator), prenatal cigarette and alcohol use by late pregnancy, child cognitive and language development, child behaviour by age 2 years, and maternal subsequent pregnancies by 24 months postpartum. ${ }^{23}$ This paper focuses on the prespecified prenatal secondary outcome indicators; we also report changes in cannabis and street drug use, which are prespecified prenatal exploratory variables. ${ }^{23}$ (All prenatal substance use measures are reported here.)

Table 1: Summary of measures at baseline and 34-36 weeks' gestation

Measurement construct and description

Scoring

Sociodemographic characteristics ${ }^{26}$

Age and cultural background (baseline only); marital status, education and income, where income was defined as pretax annual income from all sources of employment including unreported income and excluding any money received from family, friends or income assistance

Single (not married or common-law); limited education (having less than high school); low income (living on a less than $\$ 20000 / y r$ ); all dichotomous (yes/no) variables

Unstable housing

Having to move 3 or more times or experiencing homelessness (past yr).

Psychological distress

Kessler Psychological Distress Scale ${ }^{27}$; Likert scale with 10 items (e.g., "About how often did you feel hopeless?")

Experiences of violence

Child maltreatment; Childhood Trauma Questionnaire, Short Form; ${ }^{28}$ Likert scale with 28 items (e.g., "When I was growing up, I didn't have enough to eat")

Intimate partner violence (past yr); Composite Abuse Scale; ${ }^{29}$ Likert scale with 30 items (e.g., "My partner told me that I wasn't good enough"); partner was defined as husband or wife, partner, or boyfriend or girlfriend for longer than $1 \mathrm{mo}$

Prenatal substance use ${ }^{30}$

Tobacco (no. of cigarettes smoked in past $2 \mathrm{~d}$ )

Alcohol (frequency of drinking in past month)

Cannabis (frequency of using all forms of cannabis, marijuana or hashish in past mo)

Street drugs (frequency of using LSD, magic mushrooms, ecstasy, cocaine, speed, heroin or crystal methamphetamines or any combination in past mo)

Any substance (use of any or all of tobacco, alcohol, cannabis and street drugs)
Dichotomous (yes/no) variable

Moderate-to-severe anxiety or depression

Moderate-to-severe levels of neglect, physical abuse, emotional abuse and/or sexual abuse

Sum of 30 items (range, 0 [never] to 5 [daily]); a score of 3 or more was used as the criterion for exposure to intimate partner violence

Count

Frequency; dichotomous (yes/no) variable

Frequency; dichotomous (yes/no) variable

Frequency; dichotomous (yes/no) variable

Frequency; dichotomous (yes/no) variable

Note: LSD = lysergic acid diethylamide. 


\section{cmaJOPEN}

Research

In addition, we are measuring associated outcomes at multiple time points including socioeconomic status; housing instability; mental and physical health, including substance use; parenting skills; experiences of violence; and receipt of public services. ${ }^{23}$ These will be reported in future publications.

\section{Sample size calculations}

Sample size was determined on the basis of detecting clinically meaningful reductions in the primary outcome indicator, average counts of physician and other health care encounters per child for injuries by age 2 years. We initially estimated that a sample size of 1040 could detect a relative risk of 0.70 reduction (base rate of $30 \%$ in comparison group reduced to $21 \%$ in intervention group) where $\alpha=0.05$ and $\beta=0.20$ and presuming low attrition $(<5 \%)$ because of access to provincial administrative health data on child injuries. ${ }^{23}$ The expected injury base rate $(0.30$ or $30 \%)$ was based on US NFP findings. ${ }^{31}$ (We later refined these calculations on the basis of analyses of $\mathrm{BC}$ child injury data [Apr. 1, 2001 to Mar. 31, 2010] from a similar population [first births for girls and young women receiving income assistance]. Data sources included the BC Medical Services Plan for maternal subsidy status and child injury physician service events [www.popdata.bc.ca/data/health/msp] and the Discharge Abstract Database for child birth events and hospital admissions for injuries [https://www.cihi.ca/ en/discharge-abstract-database-metadata-dad]). Among 17534 children, the proportion with at least 1 injury visit (either physician encounter or hospital admission) was 0.30 (30\%). The rate of physician encounters was 239 per 1000 person years. Assuming a 30\% reduction in the rate due to the intervention (relative rate 0.70 ), the required sample size to detect a difference in the incidence rate was 349 people per study arm, plus 5\% attrition, for a total of 732 (Appendix 1B).

\section{Statistical analysis}

We conducted intention-to-treat analyses of NFP's effects on cigarette and alcohol use, on the number of cigarettes smoked (in the past $2 \mathrm{~d}$ ) for those reporting any cigarette use at baseline or 34-36 weeks' gestation or both, and on cannabis and street drug use. We analyzed the change in substance use from baseline to 34-36 weeks' gestation using generalized linear mixedeffect models (GLMMs). Specifically, we analyzed binary outcomes (cigarette, cannabis and any substance use) using logistic mixed-effect models and the count outcome (number of cigarettes used by smokers in the past $2 \mathrm{~d}$ ) using Poisson mixedeffect models. For alcohol and street drug use, the logistic mixed-effect models did not converge, so we fitted linear mixedeffect models. This approach is considered acceptable for binary outcomes when there are sufficient degrees of freedom.

The GLMMs incorporated fixed effects for time period (baseline v. 34-36 weeks' gestation); NFP versus comparison at baseline; and time period by NFP interaction for NFP intervention effect by 34-36 weeks' gestation. These models also included random effects for clusters (participants nested within local health areas within health authorities). Model estimates (95\% confidence intervals [CIs]) and associated 2 -sided $p$ values were determined. We conducted robust analyses with GLMMs adjusting additionally for all baseline covariates (Appendix 1B, Supplemental Table S1).

To evaluate marginal (population averaged) treatment effect estimates, we calculated marginal group differences (in beforeafter changes of percentages for positive binary outcomes and counts for cigarette use) using estimated regression coefficients and estimated population distribution of random effects from GLMMs. ${ }^{32}$ We obtained 95\% CIs for marginal effect estimates by repeating the marginal treatment effect estimation described above on 1000 bootstrap samples obtained from resampling at the participant level. We assessed the sensitivity to missing-atrandom assumptions via selection models. ${ }^{33-36} \mathrm{We}$ also calculated marginal group ratios (of before-after odd ratios for binary outcomes and rate ratios for count outcomes). For ease in interpretation, we report the marginal treatment effects in terms of difference in changes; coefficient estimates of mixed effects models and sensitivity analyses are also reported (Appendix 1B, Supplemental Tables S1-S3). All analyses were conducted using $\mathrm{R}$ version 3.5 (R Project for Statistical Computing).

\section{Ethics approval}

We obtained ethics approval from Simon Fraser University, the University of British Columbia, the University of Victoria, McMaster University, the Public Health Agency of Canada, Fraser Health, Interior Health, Vancouver Island Health Authority and Vancouver Coastal Health. An independent data and safety monitoring committee tracks participant safety and protocol compliance.

\section{Results}

Following baseline interviews, 739 RCT participants were randomly allocated to either the intervention group $(n=368)$ or the comparison group $(n=371) ; 667(90.2 \%)$ completed interviews at 34-36 weeks' gestation (Figure 1). All 739 participants were included in the intention-to-treat analyses.

Median gestational age at baseline was 20 weeks, 6 days. Intervention participants received a mean of 10 prenatal nursing visits, adhering to NFP fidelity guidelines. Baseline characteristics were balanced across the 2 trial groups (Table 2). (Appendix 1C, Supplemental Table S4 provides participant baseline characteristics by age group: 14-19 yr and 20-24 yr.) No protocol deviations or unanticipated serious adverse events occurred (Appendix 1D).

\section{Outcomes and effect estimation}

\section{Cigarette smoking}

At baseline, 26.6\% of participants reported smoking cigarettes (in the past 2 days). By 34-36 weeks' gestation, the proportion decreased in both groups: by $5.8 \%$ for the intervention group versus $2.5 \%$ for the comparison group (Table 3 ). The marginal difference in before-after changes (DIC) of smoking percentage was -2.8 (95\% CI -15.3 to 0.6$)$ (Table 4$)$.

We explored cigarette use for "smokers," that is, participants who used cigarettes at baseline or at 34-36 weeks' gestation or both ( $n=211 ; 28.5 \%$ of sample). By 34-36 weeks' gestation, 


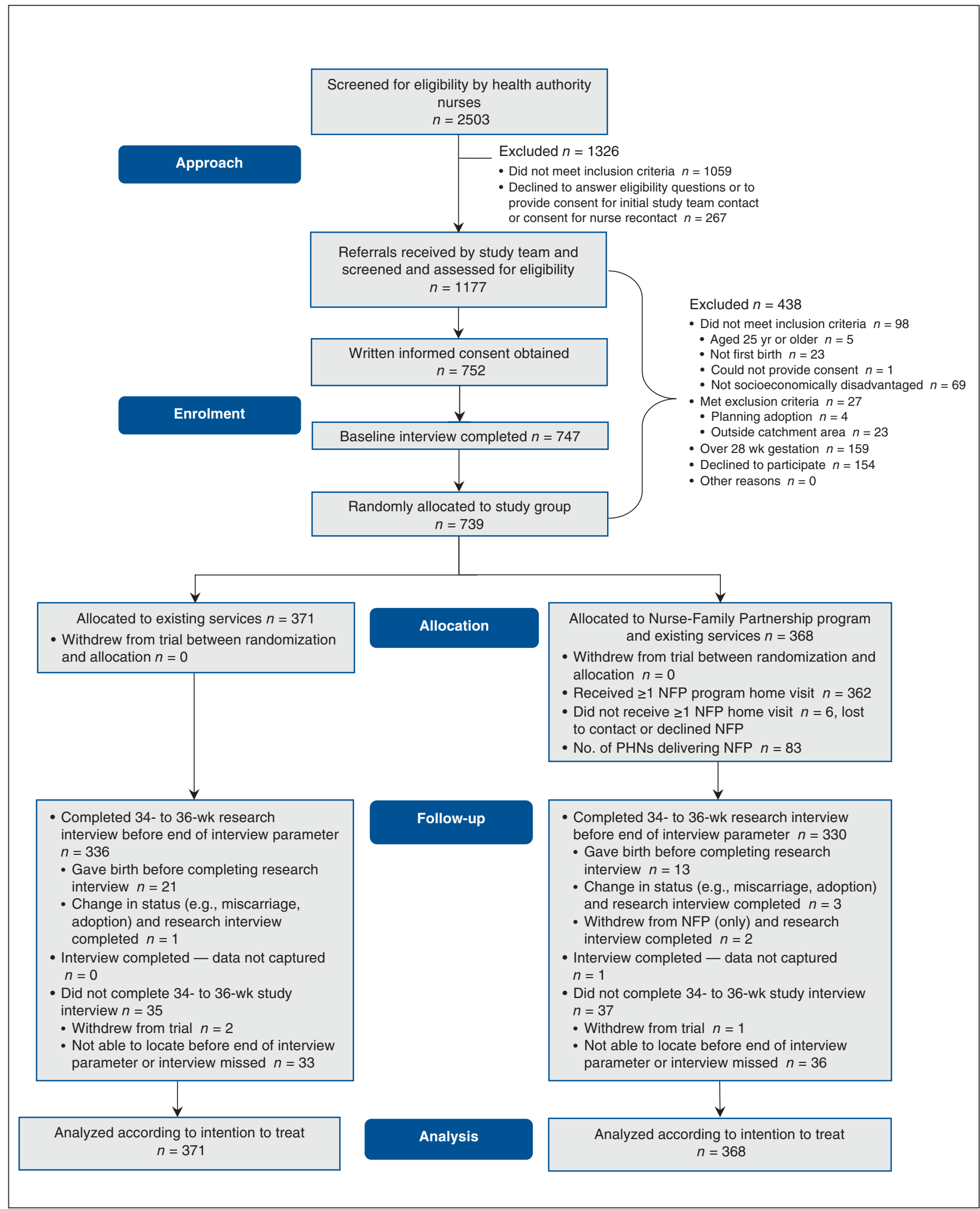

Figure 1: Participant flow diagram. Note: NFP = Nurse-Family Partnership, PHNs = public health nurses. The preallocation portion of this flow chart was originally published elsewhere ${ }^{24}$; slight wording changes have been made. The previously published information ${ }^{24}$ is used under the terms of the Creative Commons Attribution 4.0 International License (http://creativecommons.org/licenses/by/4.0/). 
Research

Table 2: Participant characteristics at baseline

\begin{tabular}{|c|c|c|}
\hline \multirow[b]{2}{*}{ Characteristic } & \multicolumn{2}{|c|}{ No. (\%) of participants; study group } \\
\hline & $\begin{array}{l}\text { Comparison } \\
n=371\end{array}$ & $\begin{array}{c}\text { NFP } \\
n=368\end{array}$ \\
\hline \multicolumn{3}{|l|}{ Sociodemographic characteristics } \\
\hline Age (19 yr or younger) & $175 / 371(47.2)$ & $186 / 368(50.5)$ \\
\hline Single (not married or common-law) & $337 / 369(91.3)$ & $333 / 367(90.7)$ \\
\hline \multicolumn{3}{|l|}{ Cultural background* } \\
\hline White & $217 / 371(58.5)$ & $201 / 368(54.6)$ \\
\hline Indigenous including First Nations, Métis and Inuit & 44/371 (11.9) & $35 / 368(9.5)$ \\
\hline $\begin{array}{l}\text { Indigenous including First Nations, Métis or Inuit and other (including } \\
\text { white or other non-Indigenous categories) }\end{array}$ & $56 / 371(15.1)$ & $65 / 368(17.7)$ \\
\hline Mixed heritage ( $\geq 2$ ethnicity categories, excluding Indigenous) & 23/371 (6.2) & $32 / 368(8.7)$ \\
\hline Asian (Chinese, South Asian or Other) & $16 / 371(4.3)$ & $16 / 368(4.3)$ \\
\hline Other (including Latin-American, Black) & $15 / 371(4.0)$ & 19/368 (5.2) \\
\hline \multicolumn{3}{|l|}{ Highest educational qualification } \\
\hline Less than high school & $193 / 371(52)$ & $191 / 367(52)$ \\
\hline High school diploma or equivalent & 139/371 (37.5) & $131 / 367(35.7)$ \\
\hline College or other nonuniversity or university degree & $39 / 371(10.5)$ & $45 / 367(12.3)$ \\
\hline \multicolumn{3}{|l|}{ Income from employment, \$ } \\
\hline$<5000$ & $146 / 362(40.3)$ & $162 / 364(44.5)$ \\
\hline $5000-9999$ & $64 / 362(17.7)$ & $54 / 364(14.8)$ \\
\hline $10000-19999$ & $87 / 362(24)$ & $93 / 364(25.5)$ \\
\hline $20000-29999$ & $38 / 362(10.5)$ & $37 / 364(10.2)$ \\
\hline$\geq 30000$ & $27 / 362(7.5)$ & $18 / 364(4.9)$ \\
\hline \multicolumn{3}{|l|}{ Unstable housing } \\
\hline Homeless ever (excluding currently) & $146 / 356(41.0)$ & $165 / 360(45.8)$ \\
\hline Currently homeless & $11 / 359(3.1)$ & $11 / 362(3.0)$ \\
\hline Moved 3 or more times or homeless (past year) & $187 / 366(51.1)$ & $198 / 365(54.2)$ \\
\hline \multicolumn{3}{|l|}{ Mental health $\dagger$} \\
\hline Moderate-to-severe psychological distress (past month) & $122 / 370(33.0)$ & $112 / 364(30.8)$ \\
\hline \multicolumn{3}{|l|}{ Experiences of violence } \\
\hline Child maltreatment at age $16 \mathrm{yr}$ or younger & 206/367 (56.1) & $204 / 361(56.5)$ \\
\hline Exposure to intimate partner violence (past year) & $187 / 369(50.7)$ & $176 / 365(48.2)$ \\
\hline \multicolumn{3}{|c|}{$\begin{array}{l}\text { Note: NFP = Nurse-Family Partnership. All measures were included as baseline covariates in the current analyses; additional reports on these measures to follow. } \\
\text { data before randomization were previously reported. }{ }^{23,24} \\
\text { "Categories according to Statistics Canada; participants could give more than } 1 \text { answer; "mixed heritage" indicates participants who self-identified with } 2 \text { or more } \\
\text { non-Indigenous categories of ethnicity. } \\
\text { †Measured according to Kessler et al. }{ }^{27}\end{array}$} \\
\hline
\end{tabular}

average cigarette counts (in the past $2 \mathrm{~d}$ ) decreased in both groups: by 2.5 for the intervention group versus 0.8 for the comparison group (Table 3). The marginal DIC of cigarette use count was -1.6 (95\% CI -6.4 to -1.3$)$ (Table 4).

\section{Alcohol use}

At baseline, 5.7\% of participants reported consuming alcohol (in the past month). By 34-36 weeks' gestation, the proportion decreased in both groups: by $2.6 \%$ for the intervention group versus $1.9 \%$ for the comparison group (Table 3 ). The marginal DIC of alcohol use percentage was -0.5 (95\% CI -8.7 to 1.8 ) (Table 4$)$.

\section{Exploratory analyses}

\section{Cannabis use}

At baseline, 25.6\% of participants reported using cannabis (in the past month). By 34-36 weeks' gestation, the proportion 
Table 3: Substance use by group and pregnancy period, before-after changes and difference in before-after changes

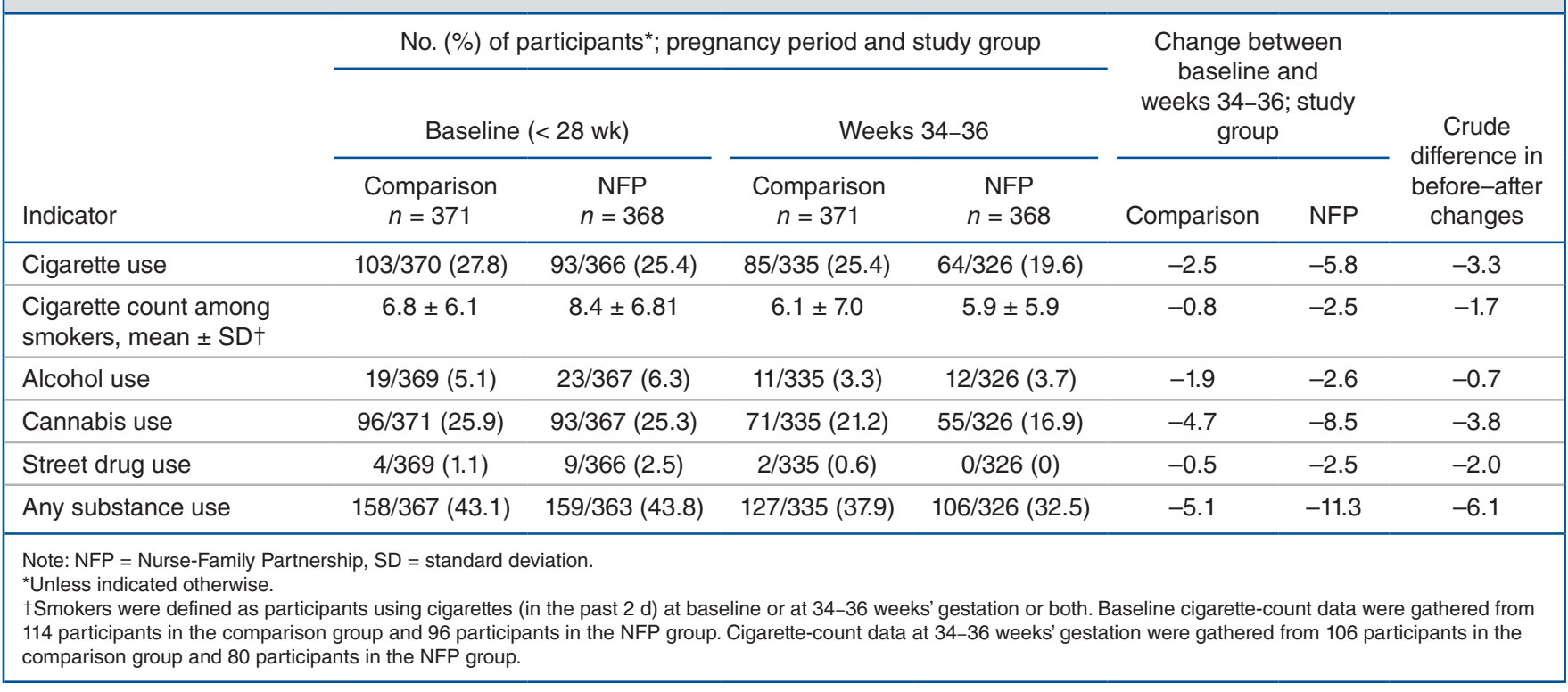

Table 4: Marginal difference in before-after changes in substance use among participants

\begin{tabular}{|lc|}
\hline Indicator & $\begin{array}{c}\text { Estimate of group } \\
\text { difference in before-after } \\
\text { changes }(95 \% \mathrm{Cl})\end{array}$ \\
\hline $\begin{array}{l}\text { Percentage of participants reporting } \\
\text { cigarette use }\end{array}$ & $-2.8(-15.3$ to 0.6$)$ \\
\hline Cigarette count among smokers & $-1.6(-6.4$ to -1.3$)$ \\
\hline $\begin{array}{l}\text { Percentage of participants reporting } \\
\text { alcohol use }\end{array}$ & $-0.5(-8.7$ to 1.8) \\
\hline $\begin{array}{l}\text { Percentage of participants reporting } \\
\text { cannabis use }\end{array}$ & $-6.4(-17.0$ to -1.7$)$ \\
\hline $\begin{array}{l}\text { Percentage of participants reporting } \\
\text { street drug use }\end{array}$ & $-2.0(-4.3$ to 1.7) \\
\hline $\begin{array}{l}\text { Percentage of participants reporting } \\
\text { any substance use }\end{array}$ & $-5.6(-13.8$ to 2.6$)$ \\
\hline $\begin{array}{l}\text { Note: Cl = confidence interval. Marginal (population averaged) estimates of } \\
\text { differences between the NFP and comparison groups (i.e., estimates of the } \\
\text { before-after changes in probability for positive binary substance outcomes and } \\
\text { rate for cigarette count) were calculated using estimated regression coefficients } \\
\text { and estimated population distribution of random effects from generalized linear } \\
\text { mixed-effect models. }\end{array}$ \\
\hline
\end{tabular}

decreased in both groups: by $8.5 \%$ for the intervention group versus $4.7 \%$ for the comparison group (Table 3). The marginal DIC of cannabis use percentage was -6.4 (95\% CI -17.0 to -1.7) (Table 4).

\section{Street drug use}

At baseline, $1.8 \%$ of participants reported using street drugs (in the past month). By 34-36 weeks' gestation, the proportion decreased in both groups: by $2.5 \%$ for the intervention group versus $0.5 \%$ for the comparison group (Table 3 ). The marginal DIC of street drug use percentage was $-2.0(95 \%$ CI -4.3 to 1.7 ) (Table 4).

\section{"Any" substance use}

At baseline, $43.4 \%$ of participants reported using "any" substance(s). By 34-36 weeks' gestation, the proportion decreased in both groups: by $11.3 \%$ for the intervention group versus $5.1 \%$ for the comparison group (Table 3 ). The marginal DIC was -5.6 (95\% CI -13.8 to 2.6) (Table 4).

\section{Sensitivity analysis}

GLMM analyses including additional baseline covariates showed that intervention effect estimates remained similar (Appendix 1B, Supplemental Table S1; last 2 columns). We found negligible impacts of nonrandom missingness on the intention-to-treat estimates (Appendix 1B, Supplemental Table S2). ${ }^{31}$

\section{Interpretation}

We report RCT data, collected prenatally, on secondary and exploratory substance use outcome indicators (all prespecified). Data on other outcome indicators will be reported in future publications.

For prenatal cigarette use, we found high baseline rates (26.6\%) but no evidence of NFP's benefit by 34-36 weeks' gestation. However, among smokers, we found significantly reduced cigarette counts for intervention participants. Similarly, NFP trials in the US and the Netherlands have shown small but important prenatal smoking reductions, although an English trial did not. ${ }^{20-22,37}$ Between-study differences may arise from divergences in populations, NFP implementation and existing health and social services. Yet while we found only 


\section{cmajOPEN}

Research

modest decreases in prenatal cigarette use among smokers, any reductions may benefit the developing fetus. ${ }^{4}$ NFP therefore shows promise, building on other harm reduction efforts. ${ }^{38-40}$

For prenatal alcohol use, we found relatively low baseline rates $(5.7 \%)$ and no evidence of benefit from NFP by 34-36 weeks' gestation. Similarly, previous US NFP trials have not shown significant reductions in prenatal alcohol use. ${ }^{20}$ As there is no safe level of prenatal alcohol consumption, ongoing harm reduction efforts remain crucial. ${ }^{5,41}$ Even so, our findings suggest that prenatal alcohol use may be too infrequent to be a useful focus for an intervention like NFP in a cohort like ours. Public health efforts to reduce prenatal drinking may also have been successful, further accounting for NFP's limited impact in our sample. ${ }^{40}$

For prenatal cannabis use, we identified elevated baseline rates $(25.6 \%){ }^{13}$ Then, by $34-36$ weeks' gestation, we found significantly reduced rates for intervention participants, which is a new finding across extant NFP trials. ${ }^{20-22,37}$ Prenatal cannabis use is rising in Canada, particularly among people in young, disadvantaged populations ${ }^{13}$ who may have greater access to cannabis than alcohol. ${ }^{42}$ These rates are concerning given the adverse consequences for the developing fetus, $1,2,6,7$ the increasing potency of cannabis ${ }^{43}$ and public perceptions of cannabis as harmless or even beneficial in pregnancy. ${ }^{44}$ Harm reduction efforts related to prenatal cannabis use should therefore be intensified. ${ }^{45}$ Our findings suggest that NFP has a role in these efforts, particularly for disadvantaged populations.

At the same time, we found no evidence of NFP's benefit regarding "any" substance use or street drug use. Similarly, for street drugs, previous NFP trials have not shown significant prenatal reductions. ${ }^{20-22}$ Low base rates for street drug use (1.8\%) also probably made it impossible to detect any effects.

\section{Limitations}

Our study has several limitations. We relied on maternal selfreport. For cigarettes in particular, self-report may underestimate smoking by up to $25 \%$ compared with serum cotinine measurements. ${ }^{46}$ Yet previous US trials using such biochemical assays have shown that compared with controls, NFP participants who were smokers became more accurate reporters by late pregnancy, giving less socially acceptable responses, potentially "disadvantaging" the intervention arm. ${ }^{47} \mathrm{We}$ collected only preliminary e-cigarette data; use has increased considerably since our trial commenced, particularly among youth. ${ }^{48}$ Future trials should examine e-cigarette use. Finally, our findings on young, disadvantaged parents-to-be in urban and suburban communities may not apply to other Canadian populations.

\section{Conclusion}

We found no evidence that NFP was effective in reducing rates of prenatal cigarette and alcohol use. However, for those who smoked, NFP led to modest but significant reductions in cigarette counts (in the past $2 \mathrm{~d}$ ). NFP also significantly reduced prenatal cannabis use, which is an emerging public health problem in Canada. Our findings therefore suggest that NFP may hold promise for reducing some types of prenatal substance use in disadvantaged populations.

\section{References}

1. Irner TB. Substance exposure in utero and developmental consequences in adolescence: a systematic review. Child Neuropsychol 2012;18:521-49.

2. Behnke M, Smith VC, Committee on Substance Abuse, et al. Prenatal substance abuse: short- and long-term effects on the exposed fetus. Pediatrics 2013; 131:e1009-24

3. Viteri OA, Soto EE, Bahado-Singh RO, et al. Fetal anomalies and long-term effects associated with substance abuse in pregnancy: a literature review. Am 7 Perinatol 2015;32:405-16.

4. Berlin I, Golmard JL, Jacob N, et al. Cigarette smoking during pregnancy: Do complete abstinence and low-level cigarette smoking have similar impact on birth weight? Nicotine Tob Res 2017;19:518-24.

5. Mamluk L, Edwards HB, Savovic J, et al. Low alcohol consumption and pregnancy and childhood outcomes: Time to change guidelines indicating apparently 'safe' levels of alcohol during pregnancy? A systematic review and metaanalyses. BMF Open 2017;7:e015410.

6. Luke S, Hutcheon J, Kendall T. Cannabis use in pregnancy in British Columbia and selected birth outcomes. 7 Obstet Gynaecol Can 2019;41: 1311-7.

7. Corsi DJ, Walsh L, Weiss D, et al. Association between self-reported prenatal cannabis use and maternal, perinatal, and neonatal outcomes. $7 A M A$ 2019;322:145-52.

8. Gouin K, Murphy K, Shah PS. Effects of cocaine use during pregnancy on low birthweight and preterm birth: systematic review and meta-analyses. $\mathrm{Am}$ 7 Obstet Gynecol 2011;204:340.e1-12.

9. Beckwith AM, Burke SA. Identification of early developmental deficits in infants with prenatal heroin, methadone, and other opioid exposure. Clin Pediatr (Phila) 2015;54:328-35

10. Cui Y, Shooshtari S, Forget EL, et al. Smoking during pregnancy: findings from the 2009-2010 Canadian Community Health Survey. PLoS One 2014;9: e84640

11. Popova S, Lange S, Probst C, et al. Prevalence of alcohol consumption during pregnancy and fetal alcohol spectrum disorders among the general and Aboriginal populations in Canada and the United States. Eur 7 Med Genet 2017;60:32-48.

12. Corsi DJ, Hsu H, Weiss D, et al. Trends and correlates of cannabis use in pregnancy: a population-based study in Ontario, Canada from 2012 to 2017. Can 7 Public Health 2019;110:76-84.

13. Porath AJ, Kent P, Konefal S. Clearing the smoke on cannabis: maternal cannabis use during pregnancy - an update. Ottawa: Canadian Centre on Substance Use and Addiction; 2018.

14. Guidelines for the identification and management of substance use and substance use disorders in pregnancy. Geneva: World Health Organization; 2014.

15. Hutson J. A prenatal perspective on the cost of substance abuse in Canada. FFAS Int 2006;4:e9-13.

16. Brown RA, Dakkak H, Gilliland J, et al. Predictors of drug use during pregnancy: the relative effects of socioeconomic, demographic, and mental health risk factors. 7 Neonatal Perinatal Med 2019;12:179-87.

17. Forray A. Substance use during pregnancy. F1000Res 2016;5:887-95.

18. Leung LW, Davies G. Smoking cessation strategies in pregnancy. 7 Obstet Gynaecol Can 2015;37:791-7.

19. Farr SL, Hutchings YL, Ondersma SJ, et al. Brief interventions for illicit drug use among peripartum women. Am 7 Obstet Gynecol 2014;211:336-43.

20. Olds DL. Preventing child maltreatment and crime with prenatal and infancy support of parents: the Nurse-Family Partnership. 7 Scand Stud Criminol Crime Prev 2008;9:2-24.

21. Mejdoubi J, van den Heijkant SCCM, van Leerdam FJM, et al. Effects of nurse home visitation on cigarette smoking, pregnancy outcomes and breastfeeding: a randomized controlled trial. Midwifery 2014;30:688-95.

22. Robling M, Bekkers MJ, Bell K, et al. Effectiveness of a nurse-led intensive home-visitation programme for first-time teenage mothers (Building Blocks): a pragmatic randomised controlled trial. Lancet 2016;387:146-55.

23. Catherine NLA, Gonzalez A, Boyle M, et al. Improving children's health and development in British Columbia through nurse home visiting: a randomized controlled trial protocol. BMC Health Serv Res 2016;16:349.

24. Catherine NLA, Lever R, Sheehan D, et al. The British Columbia Healthy Connections Project: findings on socioeconomic disadvantage in early pregnancy. BMC Public Health 2019;19:1161.

25. Jack SM, Catherine N, Gonzalez A, et al. Adapting, piloting and evaluating complex public health interventions: lessons learned from the Nurse-Family Partnership in Canadian public health settings. Health Promot Chronic Dis Prev Can 2015;35:151-9.

26. 2006 census of population. Ottawa: Statistics Canada; 2010. Available: https:// www12.statcan.gc.ca/census-recensement/2006/index-eng.cfm (accessed 2019 June 8).

27. Kessler RC, Andrews G, Colpe LJ, et al. Short screening scales to monitor population prevalences and trends in non-specific psychological distress. Psychol Med 2002;32:959-76.

28. Bernstein DP, Stein JA, Newcomb MD, et al. Development and validation of a brief screening version of the Childhood Trauma Questionnaire. Child Abuse Negl 2003;27:169-90. 
29. Hegarty K, Bush R, Sheehan M. The Composite Abuse Scale: further development and assessment of reliability and validity of a multidimensional partner abuse measure in clinical settings. Violence Vict 2005;20:529-47.

30. National Longitudinal Survey of Children and Youth (cycle 8). Ottawa: Statistics Canada; 2009. Available: http://www23.statcan.gc.ca/imdb-bmdi/ document/4450_D2_T9_V4-eng.pdf (accessed 2019 Feb. 5).

31. Olds DL, Henderson CR Jr, Chamberlin R, et al. Preventing child abuse and neglect: a randomized trial of nurse home visitation. Pediatrics 1986;78:65-78

32. Pavlou M, Ambler G, Seaman S, et al. A note on obtaining correct marginal predictions from a random intercepts model for binary outcomes. BMC Med Res Methodol 2015;15:59.

33. Ma G, Troxel AB, Heitjan DF. An index of local sensitivity to nonignorable drop-out in longitudinal modelling. Stat Med 2005;24:2129-50.

34. Xie H. A local sensitivity analysis approach to longitudinal non-Gaussian data with non-ignorable dropout. Stat Med 2008;27:3155-77.

35. Xie H, Qian Y. Measuring the impact of nonignorability in panel data with non-monotone nonresponse. F Appl Econ 2012;27:129-59.

36. Xie $\mathrm{H}$, Gao W, Xing B, et al. Measuring the impact of nonignorable missingness using the R package Isni. Comput Methods Programs Biomed 2018;164:207-20.

37. Miller TR. Projected outcomes of Nurse Family Partnership home visitation during 1996-2013, USA. Prev Sci 2015;16:765-77.

38. Greaves L, Poole N, Hemsing N. Tailored intervention for smoking reduction and cessation for young and socially disadvantaged women during pregnancy. 7 Obstet Gynecol Neonatal Nurs 2019;48:90-8.

39. Twyman L, Bonevski B, Paul C, et al. Perceived barriers to smoking cessation in selected vulnerable groups: a systematic review of the qualitative and quantitative literature. BM7 Open 2014;4:e006414.

40. Marcellus L, Poole N, Hemsing N. Beyond abstinence: harm reduction during pregnancy and early parenting. In: Peterson W, Armstrong LL, Foulkes M, editors. Mothers, addiction and recovery: finding meaning through the journey. Bradford (ON): Demeter Press; 2018.

41. Nathoo T, Poole N, Bryans M, et al. Voices from the community: developing effective community programs to support pregnant and early parenting women who use alcohol and other substances. First Peoples Child Family Rev 2013;8:93-106.

42. Singh VAS, Thornton T, Tonmyr L. Determinants of substance abuse in a population of children and adolescents involved with the child welfare system. Int 7 Ment Health Addict 2011;9:382-97.

43. Stickrath E. Marijuana use in pregnancy: an updated look at marijuana use and its impact on pregnancy. Clin Obstet Gynecol 2019;62:185-90.

44. Bayrampour H, Zahradnik M, Lisonkova S, et al. Women's perspectives about cannabis use during pregnancy and the postpartum period: an integrative review. Prev Med 2019;119:17-23.

45. Cannabis Act (S.C. 2018, c. 16).

46. Shipton D, Tappin DM, Vadiveloo T, et al. Reliability of self-reported smoking status by pregnant women for estimating smoking prevalence: a retrospective, cross sectional study. BM7 2009;339:b4347.

47. Olds DL, Henderson CR, Tatelbaum R, et al. Improving the delivery of prenatal care and outcomes of pregnancy: a randomized trial of nurse home visitation. Pediatrics 1986;77:16-28.

48. Hammond D, Reid JL, Rynard VL, et al. Prevalence of vaping and smoking among adolescents in Canada, England, and the United states: repeat national cross-sectional surveys. BM7 2019;365:I2219.

Affiliations: Children's Health Policy Centre, Faculty of Health Sciences (Catherine, Zheng, Lever, Sheehan, Cullen, Hjertaas, Riebe, Rikert, Sunthoram, Waddell), Simon Fraser University, Vancouver, BC; Offord Centre for Child Studies, Faculty of Health Sciences (Boyle, Gonzalez, Jack, MacMillan), McMaster University, Hamilton, Ont.; Faculty of Health Sciences (McCandless, Xie), Simon Fraser University, Burnaby, BC; Arthritis Research Canada (Xie), Richmond, BC; School of Nursing, Faculty of Health Sciences (Jack) and Department of Health Research Methods, Evidence and Impact, Faculty of Health Sciences (Gafni), McMaster University, Hamilton, Ont.; Public Health Agency of Canada (Tonmyr), Ottawa, Ont.; School of Nursing (Marcellus), University of Victoria, Victoria, BC; School of Nursing (Varcoe); Department of Pediatrics (Barr), Faculty of Medicine, University of British Columbia, Vancouver, BC
Contributors: All authors all made substantial contributions to the study design. Nicole Catherine oversaw the randomization allocation and ensured that NFP nurses were informed of treatment allocations. Nicole Catherine supervised all aspects of data acquisition and preparation by field interviewers (including Ange Cullen, Kathleen Hjertaas, Caitlin Riebe, Nikolina Rikert and Ashvini Sunthoram), with assistance from Rosemary Lever. Michael Boyle prepared the initial statistical analysis plan, which was later revised together with Lawrence McCandless, Hui Xie and Yufei Zheng in consultation with Nicole Catherine, Harriet MacMillan and Charlotte Waddell. Ange Cullen, Kathleen Hjertaas, Caitlin Riebe, Nikolina Rikert and Ashvini Sunthoram made substantial contributions to designing and developing the trial's daily operating procedures, acquiring the data and preparing the data for analyses as the study proceeded. Michael Boyle, Yufei Zheng, Lawrence McCandless, Hui Xie and Nicole Catherine conducted the formal data analyses and all authors interpreted the data. Nicole Catherine and Charlotte Waddell drafted the manuscript. All authors then critically revised the manuscript for important intellectual content, approved the final version, approved the subsequent revisions, and have agreed to be accountable for all aspects of the work.

Funding: This trial is funded by the British Columbia Ministry of Health with support from the British Columbia Ministry of Children and Family Development (grant XLP0764X01 Sept. 1, 2011, through Mar. 31, 2022). British Columbia's Fraser Health, Interior Health, Vancouver Island Health Authority and Vancouver Coastal Health funded the delivery of the Nurse-Family Partnership intervention. The Djavad Mowafaghian Foundation and the R. and J. Stern Family Foundation have provided additional generous supports. The opinions, results and conclusions reported in this paper are those of the authors and are independent from the funding sources. The funders were not involved in the study design; in the collection, analysis and interpretation of data; in the writing of the report; or in the decision to submit the article for publication.

Data sharing: Following completion of this trial and following publication of all major findings, anonymized participant data and associated documents including the study protocol and statistical analysis plan may be shared. Protocols for accessing study data and information will be governed by a data-sharing agreement. For more information, please contact the corresponding author.

Acknowledgements: The authors thank the families who participated in the study. This work is dedicated to them. They acknowledge the public health nurses and supervisors who delivered the intervention and the study team members who have implemented the trial. They are also grateful to their policy and practice collaborators, and to David Olds for consulting. During this study, Michael Boyle and Charlotte Waddell were supported by Canada Research Chairs. Hui Xie was supported by the Maureen and Milan Illich/Merck Chair in Statistics for Arthritis as well as by Natural Sciences and Engineering Research Council grant RGPIN-2018-04313 and National Institutes of Health grant R01CA178061 for the development of the statistical methods used in the analysis of missing data in this clinical trial. Andrea Gonzalez was supported by a Canadian Institutes of Health Research New Investigator Award and by an Ontario Ministry of Research, Innovation and Science Early Researcher Award. Harriet MacMillan was supported by the Chedoke Health Chair in Child Psychiatry.

Supplemental information: For reviewer comments and the original submission of this manuscript, please see www.cmajopen.ca/content/8/4/ E667/suppl/DC1. 